\title{
From semifield flocks to the generalized translation dual of a semifield
}

\author{
Guglielmo Lunardon*
}

\begin{abstract}
The goal of this article is to present developments of Thas' relationship between translation geneneralized quadrangles and symplectic semifield spreads. Finally we discuss some open problems.
\end{abstract}

Keywords: ovoids, semifield flocks, semifields, spreads, geometric spread sets, polar spaces

MSC 2010: 51E20, 51E15

\section{Introduction}

If $\mathcal{F}$ is a semifeld flock, the point-line dual of the associated generalized quadrangle $Q(\mathcal{F})$ is a translation quadrangle. In [33] J.A. Thas proved that the translation dual of the point-line dual of $Q(\mathcal{F})$ has subquadrangles isomorphic to $Q(4, q)$. Hence, one can construct an ovoid of $Q(4, q)$ via the general theory of generalized quadrangles. Such an ovoid defines, via the Klein correspondence, a symplectic semifield spread of $\mathrm{PG}(3, q)$.

After Thas' result, there was a renewned interest in finite semifield spreads from different points of view and his seminal ideas and constructions were generalized in different ways. Many new results have been obtained but, unfortunately, neither new semifields flocks nor the proof that new ones do not exist!

In this paper we present some developments of Thas' ideas and we discuss some open problems.

${ }^{*}$ The author is partially supported by MIUR and GNSAGA of INdAM. 


\section{Thas' results}

\subsection{Ovoids of orthogonal polar spaces}

Denote by $\mathbb{P}$ either the polar space $Q(2 n, q)(n \geq 2)$ associated with a nonsingular parabolic quadric of $\operatorname{PG}(2 n, q)$ or the polar space $Q^{+}(2 n+1, q)$ associated with a non-singular hyperbolic quadric of $\operatorname{PG}(2 n+1, q)$.

An ovoid $\mathcal{O}$ of $\mathbb{P}$ is a set of $q^{n}+1$ points no two collinear in $\mathbb{P}$. Two ovoids $\mathcal{O}_{1}$ and $\mathcal{O}_{2}$ of $\mathbb{P}$ are called isomorphic if there is an automorphism $\tau$ of $\mathbb{P}$ which maps $\mathcal{O}_{1}$ onto $\mathcal{O}_{2}$.

For $n>2$ ovoids of $\mathbb{P}$ are rare objects. Examples of ovoids of $Q^{+}(7, q)$ are known for $q$ even, for $q \equiv 2(\bmod 3)$ and for $q$ an odd prime (see $[10,31$, $4,27]$ ). For $n>3$, only some non-existence conditions are known (see [2]). Ovoids of $Q(2 n, q)$ do not exist if $n>3$ (see [9]) and $Q\left(6,2^{e}\right)$ has no ovoids (see [31]). The only two known ovoids of $Q(6, q)$ are the unitary ovoid of $Q\left(6,3^{e}\right)$ and the Ree ovoid of $Q\left(6,3^{2 e+1}\right)$ (see $[10,31]$ ), which are also ovoids of the generalized hexagon $H(q)$ of order $q$ associated with $Q(6, q)$.

We say that $\mathcal{O}$ is a translation ovoid with respect to a point $x \in \mathcal{O}$ if there is a collineation group of $\mathbb{P}$ fixing the point $x$ linewise and acting sharply transitively on $\mathcal{O} \backslash\{x\}$.

Using a non-classical model of $\mathbb{P}$, the following theorem has been proved in $[23]$.

Theorem 2.1 (Lunardon, Polverino [23]). There exists a translation ovoid of the orthogonal space $\mathbb{P}$ if and only if $\mathbb{P}$ is one of $Q^{+}(3, q), Q(4, q), Q^{+}(5, q)$.

We recall that a generalized quadrangle is a polar space of rank 2, and that $Q(4, q)$ is the classical generalized quadrangle associated with a non-singular parabolic quadric of $\mathrm{PG}(4, q)$.

\subsection{Semifield flocks}

Let $K$ be a quadratic cone of $\mathrm{PG}(3, q)$ with vertex $v$. A flock $\mathcal{F}$ of $K$ is a partition of $K \backslash\{v\}$ into $q$ conics. If all planes containing the elements of the flock $\mathcal{F}$ share a common line, then $\mathcal{F}$ is called linear. Two flocks $\mathcal{F}_{1}$ and $\mathcal{F}_{2}$ are isomorphic if there is a collineation $\tau$ of $\operatorname{PG}(3, q)$ which fixes $K$ and maps $\mathcal{F}_{1}$ onto $\mathcal{F}_{2}$.

Let $\mathcal{F}$ be a flock of the quadratic cone $K$ of $\mathrm{PG}(3, q)$ with equation $x_{2}^{2}-x_{0} x_{1}=$ 0 . Then there are two functions $f$ and $g$ of $\mathbb{F}_{q}$ to itself such that $\mathcal{F}=\left\{K \cap \pi_{t} \mid\right.$ $\left.t \in \mathbb{F}_{q}\right\}$ where $\pi_{t}$ is the plane with equation $t x_{0}-f(t) x_{1}+g(t) x_{2}+x_{3}=0$. We 
will write $\mathcal{F}=\mathcal{F}(f, g)$ and we will say that $\pi_{t}$ is a plane of the flock. We can suppose $f(0)=g(0)=0$ (see [8]).

We say that $\mathcal{F}=\mathcal{F}(f, g)$ is a semifield flock if $f, g$ are additive maps. It has been proved by N. L. Johnson in [15] that when $q$ is even, the linear flock is the only semifield flock.

By using some constructions of S.E. Payne [28] and W.M Kantor [11] of generalized quadrangles as coset geometries, the following theorem had been proved

Theorem 2.2 (J.A. Thas [32]). Given a flock $\mathcal{F}$ of a quadratic cone of $\mathrm{PG}(3, q)$, then there is a standard construction of a generalized quadrangle $Q(\mathcal{F})$ associated with $\mathcal{F}$.

The generalized quadrangle associated with a flock has a non-abelian collineation group fixing a distinguished point $(\infty)$ linewise and acting sharply transitively on the points not collinear with $(\infty)$. Moreover $Q(\mathcal{F})$ is isomorphic to the classical generalized quadrangle $H\left(3, q^{2}\right)$ if and only if $\mathcal{F}$ is linear.

When $\mathcal{F}$ is a semifield flock, then there are a line [ $\infty]$ incident with the special point $(\infty)$ and an elementary abelian group of collineations of $Q(\mathcal{F})$ of order $q^{2}$ fixing [ $\left.\infty\right]$ pointwise and acting sharply transitively on the lines of $Q(\mathcal{F})$ at distance 4 from [ $\infty$ ] (Johnson [15]). This implies that, for $q$ odd, $Q(\mathcal{F})$ is very close to be a classical quadrangle (see [36] for more details).

\subsection{Translation ovoids of $Q(4, q)$ and semifield flocks}

In this section we always suppose $q$ odd because, for $q$ even, semifield flocks are linear.

Let $C$ be a non-singular conic of $\pi=\mathrm{PG}(2, q)$. Embed $\pi$ as a plane in $\mathrm{PG}(3, q)$. Define an incidence structure $T_{2}(C)$ as follows. Points are $(i)$ the points of $\mathrm{PG}(3, q) \backslash \pi$, (ii) the planes $\alpha$ of $\mathrm{PG}(3, q)$ for which $|\alpha \cap C|=1$, and (iii) a new symbol $(\infty)$. Lines are $(a)$ the lines of $\mathrm{PG}(3, q)$ which are not contained in $\pi$ and meet $C$ (necessarily in a unique point), and (b) the points of $C$. Incidence is defined as follows. A point of type $(i)$ is incident only with lines of type $(a)$; here the incidence is that of $\mathrm{PG}(3, q)$. A point of type $(i i)$ is incident with all lines of type $(a)$ contained in it and with the unique element of $C$ in it. The point $(\infty)$ is incident with no line of type $(a)$ and all lines of type $(b)$. It is an easy exercise to show that the so defined incidence structure is a generalized quadrangle (see $[29, \S 5.3]$ ).

Denote by $Q(4, q)$ the generalized quadrangle associated with the non-singular quadric of $\operatorname{PG}(4, q)$ with equation $x_{0} x_{4}+x_{1} x_{3}+x_{2}^{2}=0$. If $\pi$ is the plane of 
$\operatorname{PG}(4, q)$ with equations $x_{0}=x_{4}=0$, then $C=\pi \cap Q(4, q)$ is a non-singular conic of $\pi$.

If $\operatorname{PG}(3, q)$ is the hyperplane of $\operatorname{PG}(4, q)$ with equation $x_{4}=0$, then $\pi$ is a plane of $\mathrm{PG}(3, q)$ and we can consider the generalized quadrangle $T_{2}(C)$ as before.

Let $\perp$ be the polarity defined by $Q(4, q)$. If $l$ and $m$ are lines of $Q(4, q)$ and $y$ is a point of $Q(4, q)$, then the map $\theta$ defined by

$$
\theta:\left\{\begin{aligned}
x=(0,0,0,0,1) & \mapsto(\infty), \\
l & \mapsto l \cap \pi, \quad \text { for } x \in l \subset x^{\perp}, \\
y \in x^{\perp} \backslash\{x\} & \mapsto y^{\perp} \cap \mathrm{PG}(3, q), \\
m \notin x^{\perp} & \mapsto\langle m,(0,0,0,0,1)\rangle \cap \operatorname{PG}(3, q), \\
\left(1, a, b, c,-b^{2}-a c\right) & \mapsto(1, a, b, c, 0),
\end{aligned}\right.
$$

is an isomorphism from $Q(4, q)$ onto $T_{2}(C)$ as generalized quadrangles.

Denote by $[a, b, c, d]$ the plane of $\mathrm{PG}(3, q)$ with equation $a x_{0}+b x_{1}+c x_{2}+$ $d x_{3}=0$. Let $\delta$ be the polarity of $\operatorname{PG}(3, q)$ defined by the map $(a, b, c, d) \mapsto$ $[a, b, c, d]$. Dualizing by $\delta$, the vertex $(0,0,0,1)$ of the cone $K$ with equation $x_{2}^{2}=x_{0} x_{1}$ is mapped to the plane $\pi$ with equation $x_{3}=0$. The $q+1$ lines of $K$ are mapped to the tangent lines of the conic $C$ of $\pi$ with equations $4 x_{0} x_{1}-x_{2}^{2}=$ $0=x_{3}$. The plane $\pi_{t}$ of the flock $\mathcal{F}(f, g)$ is mapped to the point $(t,-f(t), g(t), 1)$ which does not belong to $\pi$. As we suppose that $q$ is odd, $\mathcal{F}(f, g)$ is a flock if and only if the line of $\mathrm{PG}(3, q)$ joining $(t,-f(t), g(t), 1)$ and $(u,-f(u), g(u), 1)$, with $t \neq u$, intersects $\pi$ in an internal point of $C$. The set of points $L(\mathcal{F})=$ $\left\{(t,-f(t), g(t), 0) \mid t \in \mathbb{F}_{q}\right\}$ is called the dual flock of $\mathcal{F}$.

Let $\mathcal{F}=\mathcal{F}(f, g)$ be a semifield flock, and let $\mathbb{F}_{s}$ be a subfield of $\mathbb{F}_{q}$ for which $f$ and $g$ are $\mathbb{F}_{s}$-linear maps of $\mathbb{F}_{q}$ to itself. If $q=s^{n}$, denote by $\bar{f}$ and $\bar{g}$ the adjoint maps of $f$ and $g$ (respectively) with respect to the $\mathbb{F}_{s}$-bilinear form defined by $\langle x, y\rangle=\operatorname{Tr}_{\mathbb{F}_{s}}(x y)$ for all $x, y \in \mathbb{F}_{q}$. Let

$$
\Omega=\left\{\left(\frac{\bar{f}(x)-\bar{g}(y)}{2}, x, y, 0\right) \mid x, y \in \mathbb{F}_{q}\right\} .
$$

If $\perp$ is the polarity of $\mathrm{PG}(3 n-1, s)$ defined by the bilinear form

$$
b\left(\left(x_{0}, x_{1}, x_{2}\right),\left(y_{0}, y_{1}, y_{2}\right)\right)=\operatorname{Tr}_{\mathbb{F}_{s}}\left(2 x_{0} y_{1}+2 x_{1} y_{0}+x_{2} y_{2}\right),
$$

then, by field reduction, $L(\mathcal{F})$ defines an $(n-1)$-subspace $U$ of $\mathrm{PG}(3 n-1, s)$ and $\Omega$ defines a $(2 n-1)$-subspace $T$ of $\mathrm{PG}(3 n-1, s)$ such that $T=U^{\perp}$. Therefore, as all points of $L(\mathcal{F})$ are internal points of $C, \Omega$ is disjoint from $C$. 
Denote by $T_{2}(C)$ the model of $Q(4, q)$ from $C$. If

$$
T(\mathcal{F})=\left\{(-\bar{f}(x)-\bar{g}(y), x, y, 1) \mid x, y \in \mathbb{F}_{q}\right\} \cup\{(\infty)\},
$$

then any line of $\operatorname{PG}(3, q)$ joining two points of $T(\mathcal{F})$ is disjoint from $C$, i.e. it is not a line of $T_{2}(C)$. As $T(\mathcal{F})$ contains $q^{2}+1$ points of $T_{2}(C)$, it is an ovoid of $T_{2}(C)$ ([34]). As $T_{2}(C)$ is isomorphic to $Q(4, q)$, we have the following theorem.

Theorem 2.3 (J.A. Thas $[33,34]) . T(\mathcal{F})$ is an ovoid of $Q(4, q)$.

Let $f(\gamma)=b \gamma$ and $g(\gamma)=a \gamma$ where $X^{2}+a X+b$ is irreducible over $\mathbb{F}_{q}$, $q$ any prime power. Then $\mathcal{F}=\mathcal{F}(f, g)$ is the linear flock, and $T(\mathcal{F})$ is an elliptic quadric.

Let $f(\gamma)=n \gamma^{\sigma}$ and $g(\gamma)=0$ where $\sigma \in \operatorname{Aut} \mathbb{F}_{q}, \sigma \neq \mathrm{id}$, and $n$ is a non square in $\mathbb{F}_{q}$. Then $\mathcal{F}=\mathcal{F}(f, g)$ is the Kantor flock (see [11, 8]), and $T(\mathcal{F})$ is the Kantor-Knuth ovoid.

Let $q=3^{r}(r \geq 3)$ and let $f(\gamma)=n \gamma^{9}+n^{-1} \gamma$ and $g(\gamma)=\gamma^{3}$. Then $\mathcal{F}=$ $\mathcal{F}(f, g)$ is the Ganley semifield flock $([7,8])$ and the ovoid $T(\mathcal{F})$ is not isomorphic to an elliptic quadric nor to the Kantor-Knuth ovoid (Payne-Thas [35]).

Analyzing the Thas' construction of $T(\mathcal{F})$ we can prove the following theorem.

Theorem 2.4 (Lunardon [18]). If $\mathcal{F}$ is a semifield flock, then $T(\mathcal{F})$ is a translation ovoid of $Q(4, q)$.

If $\mathcal{O}$ is a translation ovoid of $Q(4, q)$ then there is a semifield flock $\mathcal{F}$ such that $\mathcal{O}=T(\mathcal{F})$.

If $\mathcal{F}_{1}$ and $\mathcal{F}_{2}$ are semifield flocks of $K$, then $\mathcal{F}_{1}$ is isomorphic to $\mathcal{F}_{2}$ if and only if $T\left(\mathcal{F}_{1}\right)$ and $T\left(\mathcal{F}_{2}\right)$ are isomorphic.

The correspondence between semifield flocks and translation ovoids was used by Bader, Lunardon and Pinneri in [1] to construct the sporadic semifield flock $\mathcal{F}$ of order 243 starting from the translation ovoid $\mathcal{O}$ of $Q\left(4,3^{5}\right)$ found by Penttila and Williams in [30]. The sporadic semifield flock $\mathcal{F}$ is not isomorphic to any of the previously known semifield flocks and it has been proved in [1] that $\mathcal{F}=\mathcal{F}(f, g)$ where $f(\gamma)=\gamma^{9}$ and $g(\gamma)=\gamma^{27}$.

No more semifield flocks are known. 


\section{A step further}

\subsection{Spreads}

A spread of $\mathrm{PG}(2 n-1, q)$ is a partition $\mathcal{S}$ of the point-set of $\mathrm{PG}(2 n-1, q)$ into $(n-1)$-dimensional subspaces. With any spread $\mathcal{S}$ is associated a translation plane $A(\mathcal{S})$. A spread $\mathcal{S}$ of $\mathrm{PG}(2 n-1, q)$ is said to be Desarguesian if $A(\mathcal{S})$ is isomorphic to $A G\left(2, q^{n}\right)$ (see, e.g., [5, $\left.\S 5.1\right]$ or [16]).

Two spreads $\mathcal{S}$ and $\mathcal{S}^{\prime}$ are isomorphic if there is a collineation $\tau$ of $\mathrm{PG}(2 n-$ $1, q$ ) such that $\mathcal{S}^{\prime}=\mathcal{S}^{\tau}$. Isomorphic spreads define isomorphic translation planes.

We say that the spread $\mathcal{S}$ is symplectic if there is a symplectic polarity of $\mathrm{PG}(2 n-1, q)$ such that all elements of $\mathcal{S}$ are totally isotropic with respect to the polarity.

A spread $\mathcal{S}$ is a semifield spread (with respect to a fixed element $A$ of $\mathcal{S}$ ) if there is a group of collineations of $\mathrm{PG}(2 n-1, q)$ fixing $A$ pointwise and acting sharply transitively on $\mathcal{S} \backslash\{A\}$. This is equivalent to saying that the plane $A(\mathcal{S})$ belongs to the Lenz-Barlotti class $\mathrm{V}$.

\subsection{Ovoids of $Q^{+}(5, q)$ and spreads of $\mathrm{PG}(3, q)$}

Let $x_{0} x_{5}+x_{1} x_{4}+x_{2} x_{3}=0$ be the equation of the Klein quadric $Q^{+}(5, q)$ of $\mathrm{PG}(5, q)$. If $l$ is the line of $\mathrm{PG}(3, q)$ joining the points $\left(x_{0}, x_{1}, x_{2}, x_{3}\right)$ and $\left(y_{0}, y_{1}, y_{2}, y_{3}\right)$, we set $p_{i j}=x_{i} y_{j}-x_{j} y_{i}$. Then the line $l$ is mapped to the point $k(l)=\left(p_{01}, p_{02}, p_{03}, p_{21}, p_{13}, p_{32}\right)$ of $Q^{+}(5, q)$. The map $k: l \mapsto k(l)$ from the lineset of $\mathrm{PG}(3, q)$ to the pointset of $Q^{+}(5, q)$ is known as the Klein correspondence. We note that the point $(1, a, b, c, d,-a d-b c)$ is the image of the line $l=\langle(1,0, c,-d),(0,1, a, b)\rangle$ of $\mathrm{PG}(3, q)$.

We recall that an ovoid of $Q^{+}(5, q)$ is a set $\mathcal{O}$ of $q^{2}+1$ points of $Q^{+}(5, q)$ no two collinear on $Q^{+}(5, q)$.

It is well known (see, e.g., [13]) that:

- a set $\mathcal{S}$ of lines of $\operatorname{PG}(3, q)$ is a spread if and only if $\mathcal{O}=k(\mathcal{S})$ is an ovoid of $Q^{+}(5, q)$;

- the spread $\mathcal{S}$ is symplectic if and only if there is a hyperplane $H$ of $\operatorname{PG}(5, q)$ containing $\mathcal{O}=k(\mathcal{S})$, i.e. $\mathcal{O}$ is an ovoid of $Q(4, q)=H \cap Q^{+}(5, q)$;

- a set $\mathcal{R}$ of $q+1$ lines of $\operatorname{PG}(3, q)$ is a regulus (i.e. a ruling of a hyperbolic quadric of $\mathrm{PG}(3, q))$ if and only if $k(\mathcal{R})$ is a conic intersection of $Q^{+}(5, q)$ with a plane; 
- a spread $\mathcal{S}$ is Desarguesian if and only if $\mathcal{O}=k(\mathcal{S})$ is an elliptic quadric contained in a 3-dimensional subspace $T$ of $\mathrm{PG}(5, q)$, i.e. $\mathcal{O}=T \cap Q^{+}(5, q)$.

An ovoid $\mathcal{O}$ of $Q^{+}(5, q)$ is a translation ovoid (with respect to a point $x \in \mathcal{O}$ ) if and only if there is a subgroup $G \subset \operatorname{PGL}(6, q)$ of order $q^{2}$ fixing $Q^{+}(5, q)$, fixing all the lines of $Q^{+}(5, q)$ incident with $x$ and acting sharply transitively on $\mathcal{O} \backslash\{x\}$.

If $q=2^{h}$ is even, translation ovoids of $Q(4, q)$ are elliptic quadrics ([3]), i.e. the desarguesian spread is the only symplectic semifield spread of $\operatorname{PG}\left(3,2^{h}\right)$.

Associated with a flock of the quadratic cone is a spread of $\mathrm{PG}(3, q)$ as independently proved by M. Walker and J.A. Thas (see [37, 6]). This spread is defined in the following way.

Let the cone $K=\Sigma \cap Q^{+}(5, q)$, where $\Sigma$ is a 3-dimensional subspace of $\operatorname{PG}(5, q)$. Let $\mathcal{F}=\left\{C_{t} \mid t \in \mathbb{F}_{q}\right\}$ be a flock of $K$; denote the plane of the conic $C_{t}$ by $\pi_{t}$ and let $\pi_{t}^{*}$ be the polar plane of $\pi_{t}$ with respect to $Q^{+}(5, q)$. Hence $C_{t}^{*}=\pi_{t}^{*} \cap Q^{+}(5, q)$ is a non-singular conic through the vertex $v$ of the cone and

$$
\mathcal{O}_{\mathcal{F}}=\cup_{t \in \mathbb{F}_{q}} C_{t}^{*}
$$

is an ovoid of $Q^{+}(5, q)$, which is the union of $q$ conics which share the vertex $v$ of $K$ (see $[37,6]$ ).

Note that all the planes $\pi_{t}^{*}$ contain the polar line of $\Sigma$ with respect to $Q^{+}(5, q)$, which is tangent to $C_{t}^{*}$ at $v$.

Theorem 3.1 (Gevaert, Johnson [8]). For each ovoid $\mathcal{O}$ of $Q^{+}(5, q)$, which is the union of $q$ conics sharing a point, there is a flock $\mathcal{F}$ of $K$ such that $\mathcal{O}$ is isomorphic to $\mathcal{O}_{\mathcal{F}}$.

The flocks $\mathcal{F}_{1}$ and $\mathcal{F}_{2}$ are isomorphic if and only if the ovoids $\mathcal{O}_{\mathcal{F}_{1}}$ and $\mathcal{O}_{\mathcal{F}_{2}}$ are.

Let $S(\mathcal{F})$ be the spread of $\mathrm{PG}(3, q)$ defined via the Klein correspondence by the ovoid $\mathcal{O}_{\mathcal{F}}$. As a conic of $Q^{+}(5, q)$ is mapped to a regulus of $\operatorname{PG}(3, q)$, then we have proved the following corollary.

Corollary 3.2 (Gevaert, Johnson [8]). For any spread $\mathcal{S}$ of $\mathrm{PG}(3, q)$ there is a flock $\mathcal{F}$ of $K$ such that $\mathcal{S}$ is isomorphic to $S(\mathcal{F})$ if and only if $\mathcal{S}$ is the union of $q$ reguli sharing a line.

Let $\mathcal{O}$ be the ovoid of $Q^{+}(5, q)$ defined (under the Klein correspondence) by the spread $\mathcal{S}$ of $\mathrm{PG}(3, q)$. By possibly changing coordinates in $\mathrm{PG}(3, q)$, we can suppose that $A=\left\{(0,0, c, d) \mid c, d \in \mathbb{F}_{q},(c, d) \neq(0,0)\right\}$ and $B=\{(a, b, 0,0) \mid$ $\left.a, b \in \mathbb{F}_{q},(a, b) \neq(0,0)\right\}$ are lines of $\mathcal{S}$. Hence the points $k(A)=(0,0,0,0,0,1)$ and $k(B)=(1,0,0,0,0,0)$ belong to $\mathcal{O}$. 
A point $x \in \mathcal{O}$ different from $k(A)$ has coordinates $(1, a, b, c, d,-a d-b c)$ and, under the Klein correspondence, it is the image of the line

$$
D=\langle(1,0, c,-d),(0,1, a, b)\rangle
$$

of $\mathcal{S}$. If $J_{D}=\left(\begin{array}{cc}c & -d \\ a & b\end{array}\right)$, then $D=\left\{\left((u, v),(u, v) J_{D}\right) \mid u, v \in \mathbb{F}_{q},(u, v) \neq(0,0)\right\}$. The set $\mathbb{C}=\left\{J_{D} \mid D \in \mathcal{S}, D \neq A\right\}$ has the following properties:

(1) $|\mathbb{C}|=q^{2}$,

(2) the zero matrix $O=J_{B}$ belongs to $\mathbb{C}$,

(3) if $X$ and $Y$ are different elements of $\mathbb{C}$, then $X-Y$ is non-singular.

Then each non-zero matrix of $\mathbb{C}$ is non-singular and $\mathbb{C}$ is called a spread set associated to $\mathcal{S}$ with respect to $A, B$. We note that $\mathbb{C}$ is not uniquely defined.

Suppose that $\mathbb{C}$ is closed under addition. For each element $X$ of $\mathbb{C}$, let $\tau_{X}$ be the collineation of $\operatorname{PG}(3, q)$ defined by the matrix $\left(\begin{array}{cc}I & X \\ O & I\end{array}\right)$ where $O$ and $I$ are respectively the zero and the identity $2 \times 2$ matrix. Then $\tau_{X}$ fixes all points of $A$ and all planes through $A$. As $\mathbb{C}$ is closed under addition, $\tau_{X}$ fixes the spread $\mathcal{S}$ and $\left\{\tau_{X} \mid X \in \mathbb{C}\right\}$ is a group of order $q^{2}$ acting sharply transitively on $\mathcal{S} \backslash\{A\}$. As a spread is a semifield spred if and only if a corresponding spread set is closed underthe addition (see, e.g., [5]), the following theorem has been proved.

Theorem 3.3 (Lunardon [21]). A spread $\mathcal{S}$ is a semifield spread if and only if $k(\mathcal{S})$ is a translation ovoid.

\subsection{Linear sets}

Let $\mathrm{PG}\left(r-1, q^{t}\right)=\mathrm{PG}\left(V, \mathrm{GF}\left(q^{t}\right)\right)$. A set $\Omega$ of points of $\mathrm{PG}\left(r-1, q^{t}\right)$ is an $\mathbb{F}_{q}$-linear set of $\mathrm{PG}\left(r-1, q^{t}\right)$ if there is a subset $W$ of $V$, which is an $\mathbb{F}_{q}$-vector subspace of $V$, such that a point of $\mathrm{PG}\left(r-1, q^{t}\right)$ belongs to $\Omega$ if and only if it is defined by a vector of $W$.

Two $\mathbb{F}_{q}$-linear sets $\Omega_{1}$ and $\Omega_{2}$ of $\mathrm{PG}\left(r-1, q^{t}\right)$ are equivalent if there is a collineation $\omega$ of $\mathrm{PG}\left(r-1, q^{t}\right)$ such that $\Omega_{1}^{\omega}=\Omega_{2}$.

If $W$ is an $\mathbb{F}_{q}$-vector space of rank $m$, we say that $\Omega$ has rank $m$.

If we regard $V$ as a vector space over $\mathbb{F}_{q}$ of rank $r t$, then $\operatorname{PG}\left(V, \mathbb{F}_{q}\right)=\operatorname{PG}(r t-$ $1, q)$. A subspace $\langle x\rangle$ of $V$ of rank 1 over $\mathbb{F}_{q^{t}}$ defines an $\mathbb{F}_{q}$-subspace $L(x)$ of rank $t$ of $V$ as a vector space over $\mathbb{F}_{q}$. Thus

$$
\mathcal{S}=\left\{L(x) \mid\langle x\rangle \text { is a point of } \mathrm{PG}\left(r-1, q^{t}\right)\right\}
$$

defines a partition of the point-set of $\mathrm{PG}(r t-1, q)$ in $(t-1)$-dimensional subspaces, called the $\mathbb{F}_{q}$-linear representation of $\mathrm{PG}\left(r-1, q^{t}\right)$. The following theorem has been proved. 
Theorem 3.4 (Lunardon $[19,20]$ ). For any $\mathbb{F}_{q}$-linear set $\Omega$ of $\Lambda=\mathrm{PG}\left(r-1, q^{t}\right)$ of rank $h$, there is an $(h-1)$-dimensional subspace $U$ of $\Sigma=\mathrm{PG}(r t-1, q)$ such that $\Omega=\{\langle x\rangle \in \Lambda \mid L(x) \cap U \neq \emptyset\}$.

Denote by $\omega$ the polarity of $\mathrm{PG}\left(r-1, q^{t}\right)$ defined by a non-singular bilinear form $\mathbf{b}$ of $V$. If $\operatorname{Tr}_{\mathbb{F}_{q}}$ is the trace of $\mathbb{F}_{q^{t}}$ over $\mathbb{F}_{q}$, the bilinear form $\operatorname{Tr}(\mathbf{b}(x ; y))$ on $V$, as a vector space over $\mathbb{F}_{q}$, defines a polarity $\perp$ of $\mathrm{PG}(r t-1, q)$.

Let $\Omega$ be an $\mathbb{F}_{q}$-linear set of rank $h$ of $\mathrm{PG}\left(r-1, q^{t}\right)$. If $U$ is a $(h-1)$-dimensional subspace of $\mathrm{PG}(r t-1, q)$ such that $\Omega=\left\{\langle x\rangle \in \mathrm{PG}\left(r-1, q^{t}\right) \mid L(x) \cap U \neq \emptyset\right\}$, and $U^{\perp}$ is the polar space of $U$ with respect to $\perp$, then $\Omega^{*}=\left\{\langle x\rangle \in \mathrm{PG}\left(r-1, q^{t}\right) \mid\right.$ $\left.L(x) \cap U^{\perp} \neq \emptyset\right\}$ is an $\mathbb{F}_{q}$-linear set of rank $r t-h$ of $\mathrm{PG}\left(r-1, q^{t}\right)$. We call $\Omega^{*}$ the dual of $\Omega$. We note that $\Omega^{*}$ does not depend, up to collineations, on the chosen polarity of $\mathrm{PG}\left(r-1, q^{t}\right)$.

\subsection{Translation ovoids of $Q^{+}(5, q)$}

As before, let $Q^{+}(5, q)$ be the polar space defined by the equation $x_{0} x_{5}+x_{1} x_{4}+$ $x_{2} x_{3}=0$ in $\mathrm{PG}(5, q)$.

Let $\Sigma^{\prime}=\mathrm{PG}(4, q)$ be the hyperplane of $\mathrm{PG}(5, q)$ with equation $x_{5}=0$ and let $\Sigma$ be the 3-dimensional subspace with equations $x_{0}=x_{5}=0$. Then $Q^{+}(3, q)=$ $\Sigma \cap Q^{+}(5, q)$ is a non-singular hyperbolic quadric of $\Sigma$. Define a point-line geometry $\mathbb{H}$ in the following way. The points are (i) a symbol $(\infty)$, (ii) the 3dimensional subspaces of $\Sigma^{\prime}$ which intersect $\Sigma$ in a plane tangent to $Q^{+}(3, q)$ and (iii) the points of $\Sigma^{\prime} \backslash \Sigma$. The lines are (I) the points of $Q^{+}(3, q)$ or (II) the lines of $\Sigma^{\prime}$ which intersect $\Sigma$ in a point of $Q^{+}(3, q)$ and (III) the planes of $\Sigma^{\prime}$ which intersect $\Sigma$ in a line of $Q^{+}(3, q)$. The point $(\infty)$ is incident only with the lines of type (I). All other incidences are inherited from $\Sigma^{\prime}$.

Let $\perp$ be the polarity defined by $Q^{+}(5, q)$. If $l$ and $m$ are lines of $Q^{+}(5, q)$ and $y$ is a point of $Q^{+}(5, q)$, then the map $\theta$ defined as

$$
\theta:\left\{\begin{aligned}
x=(0,0,0,0,0,1) & \mapsto(\infty), \\
l & \mapsto l \cap \Sigma, \quad \text { for } x \in l \subset x^{\perp}, \\
l & \mapsto l^{\perp} \cap \Sigma^{\prime}, \quad \text { for } x \notin l \subset x^{\perp}, \\
y \in x^{\perp} \backslash\{x\} & \mapsto y^{\perp} \cap \Sigma^{\prime}, \\
m \not \subset x^{\perp} & \mapsto\langle m,(0,0,0,0,0,1)\rangle \cap \Sigma^{\prime}, \\
(1, b, c, d, e,-b e-c d) & \mapsto(1, b, c, d, e, 0),
\end{aligned}\right.
$$

is an isomorphism from $Q^{+}(5, q)$ onto $\mathbb{H}$. The group $E$ of all elations of $\Sigma^{\prime}$ with axis $\Sigma$ defines a translation group of $\mathbb{H}$ with base point $(\infty)$, i.e. the group of all 
collineations of $\mathbb{H}$ fixing the point $(\infty)$ linewise. We note that $E$ has a canonical structure of $\mathbb{F}_{q}$-vector space.

Let $\alpha$ be a plane of $\Sigma$ such that $\alpha \cap Q^{+}(3, q)=C$ is a non-singular conic. If $\Lambda$ is a hyperplane of $\Sigma^{\prime}=\operatorname{PG}(4, q)$ such that $\Lambda \cap \Sigma=\alpha$, then the point-line geometry $\mathbb{H}_{\alpha}$, whose elements are either $(\infty)$ or the subspaces of $\Lambda$, which are the intersection of elements of $\mathbb{H}$ with $\Lambda$, is canonically isomorphic via $\theta$ to the polar space

$$
Q(4, q)=Q^{+}(5, q) \cap\langle(0,0,0,0,0,1), \Lambda\rangle .
$$

We note that $\mathbb{H}_{\alpha}$ is the generalized quadrangle $T_{2}(C)$ (see [29]).

As in Section 3.2, denote by $k(A)$ the point of $Q^{+}(5, q)$ associated with the line $A$ of $\mathrm{PG}(3, q)$ under the Klein correspondence.

If $\mathcal{O}$ is an ovoid of $Q^{+}(5, q)$ containing the point $(0,0,0,0,0,1)$, let $\mathfrak{p}(\mathcal{O})$ be the projection of $\mathcal{O}^{\theta}$ from $(1,0,0,0,0,0)=k(B)^{\theta}$ to $\Sigma$.

If $\mathcal{F}=\mathcal{F}(f, g)$ is a semifield flock, $\mathcal{S}=S(\mathcal{F})$ is the semifield spread associated with $\mathcal{F}$ and $\mathcal{O}=k(\mathcal{S})$, then

$$
\mathfrak{p}(\mathcal{O})=\left\{(0, t, u, u+g(t),-f(t), 0) \mid t, u \in \mathbb{F}_{q}\right\},
$$

i.e. $\mathfrak{p}(\mathcal{O})$ is union of lines through the point $(0,0,1,1,0,0)$. As a first result we can restate the characterisation of flock spreads by Gevaert and Johnson.

Theorem 3.5 (Lunardon [21]). Let $\mathcal{O}$ be an ovoid of $\mathbb{H}$ containing the point $(\infty)$. There is a flock $\mathcal{F}$ such that $\mathcal{O}=\mathcal{O}_{\mathcal{F}}$ if and only if $\mathfrak{p}(\mathcal{O})$ is union of lines through a common point.

Let $\mathcal{O}$ be an ovoid of $Q^{+}(5, q)$. If $\mathcal{O}=k(\mathcal{S})$ and $\mathbb{C}$ is a spread set with respect to $A, B$ associated with $\mathcal{S}$ then, changing the homogeneous coordinates in $\mathrm{PG}(3, q)$ (if necessary, cf. Section 3.2) one can suppose $k(A)=(0,0,0,0,0,1)$, $k(B)=(1,0,0,0,0,0)$. If $J_{D}=\left(\begin{array}{cc}c & -d \\ a & b\end{array}\right)$, then the line

$$
D=\langle(1,0, c,-d),(0,1, a, b)\rangle
$$

is represented on the Klein quadric by the point $k(D)=(1, a, b, c, d,-c b-a d)$ and $k(D)^{\theta}=(1, a, b, c, d, 0)$. Hence

$$
\mathfrak{p}(\mathcal{O})=\left\{(0, a, b, c, d, 0) \mid J_{D}=\left(\begin{array}{cc}
c & -d \\
a & b
\end{array}\right) \in \mathbb{C} ; J_{D} \neq 0\right\} .
$$

If $\mathcal{S}$ is a semifield spread, then $\mathbb{C}$ is closed under summation. Therefore there is a subfield $\mathbb{F}_{s}$ of $\mathbb{F}_{q}\left(q=s^{n}\right)$, such that $\mathbb{C}$ is an $\mathbb{F}_{s}$-vector space of rank $2 n$. With the above notation, the following holds. 
Theorem 3.6 (Lunardon [21]). Let $\mathcal{O}$ be an ovoid of $\mathbb{H}$ containing the points $(1,0,0,0,0,0)$ and $(\infty)$ and let $\mathcal{S}$ be the spread of $\mathrm{PG}(3, q)$ such that $k(\mathcal{S})^{\theta}=\mathcal{O}$. The spread $\mathcal{S}$ is a semifield spread if and only if $\Omega=\mathfrak{p}(\mathcal{O})$ is an $\mathbb{F}_{s}$-linear set of rank $2 n$ disjoint from the quadric $Q^{+}(3, q)=\Sigma \cap Q^{+}(5, q)$.

The above theorem, obtained as byproduct while studing the relationship between semifield flocks and symplectic spreads, plays an important role in the theory of the planes of Lenz-Barlotti class V. Indeed, it was a widespread belief (see, e.g., [38]) that it would be possible to classify all finite planes of class V, but using such a construction in Theorem 3.6, many new examples have been exhibited by different authors (S. Ball, I. Cardinali, G.L. Ebert, N.L. Johnson, M. Lavrauw, G. Marino, O. Polverino, J. Sheekey, R. Trombetti,...) disproving the above conjecture (for more details see, e.g., [22]).

If $\perp$ is the polarity of $\mathrm{PG}(3, q)$ associated with $Q^{+}(3, q)$, let $\Omega^{\perp}$ be the dual of $\Omega$ with respect to $\perp$. Then $\Omega^{\perp}$ is disjoint from $Q^{+}(3, q)$ too, hence it defines a new semifield spread of $\mathrm{PG}(3, q)$, denoted $\mathcal{S}^{\perp}$, called the translation dual of $\mathcal{S}$.

Theorem 3.7 (Lunardon [21]). Two semifield spreads $\mathcal{S}_{1}$ and $\mathcal{S}_{2}$ of $\mathrm{PG}(3, q)$ are isomorphic if and only if $\mathcal{S}_{1}^{\perp}$ and $\mathcal{S}_{2}^{\perp}$ are isomorphic.

As a consequence of this result we have that the translation dual operation is "well defined".

We note that $\Omega^{\perp}$ is contained in a plane if and only if $\Omega$ is contained in a pencil of lines. Hence, by Theorem 3.5, we can restate the original result of J.A. Thas.

Corollary 3.8. The semifield spread $\mathcal{S}$ is union of $q$ reguli which share a line (i.e. $\mathcal{S}$ is the spread associated with a semifield flock of the quadratic cone of $\mathrm{PG}(3, q)$ ) if and only if $\mathcal{S}^{\perp}$ is a symplectic spread of $\mathrm{PG}(3, q)$.

\section{Recent steps}

\subsection{Geometric spread sets and semifields}

In the previous section, using the Klein correspondence between the lines of $\mathrm{PG}(3, q)$ and the points of $Q^{+}(5, q)$, we studied the semifield spreads of $\mathrm{PG}(3, q)$ as translation ovoids of $Q^{+}(5, q)$ but this point of view is not anymore possible for semifield spreads of $\mathrm{PG}(2 n-1, q)(n>2)$. Hence we have used a different approach, more algebraic than the previous one.

A presemifield is an algebraic structure satisfying all the axioms for a skewfield except (possibly) associativity. A presemifield is a semifield if there is an identity element. 
Let $E=\operatorname{End}\left(\mathbb{F}_{q^{n}}, \mathbb{F}_{q}\right)$ be the vector space of all the endomorphisms of $\mathbb{F}_{q^{n}}$ as vector space over $\mathbb{F}_{q}$. The Segre variety $S_{n, n}$ of the projective space $\mathrm{PG}\left(n^{2}-1, q\right)=\mathrm{PG}\left(E, \mathbb{F}_{q}\right)$ is the algebraic variety defined by the elements of $E$ of rank 1 and the $(n-2)$-secant variety $\mathcal{M}\left(S_{n, n}\right)$ (the secant variety, for short) of $S_{n, n}$ is the hypersurface of $\mathrm{PG}\left(n^{2}-1, q\right)$ defined by the singular elements of $E$.

The Segre variety has two rulings of $(n-1)$-dimensional subspaces, say $\mathcal{R}$ and $\mathcal{R}^{\prime}$, that satisfy the following properties:

(a) the subspaces of $\mathcal{R}$ (resp. $\mathcal{R}^{\prime}$ ) are mutually disjoint;

(b) if $X \in \mathcal{R}$ and $X^{\prime} \in \mathcal{R}^{\prime}$ then $X \cap X^{\prime}$ is a point;

(c) each point of $S_{n, n}$ belongs to a unique element of $\mathcal{R}$ and to a unique element of $\mathcal{R}^{\prime}$.

For more details see [14, Section 25.5].

An $\mathbb{F}_{s}$-linear set $\Omega$ of $\mathrm{PG}\left(n^{2}-1, q\right)$ of rank $n r\left(q=s^{r}\right)$ is called a geometric spread set if $\Omega \cap \mathcal{M}\left(S_{n, n}\right)=\emptyset$. By definition of linear set, there is an $\mathbb{F}_{s}$-vector subspace of rank $r n$, say $\mathbb{C}$, of $E$ such that $\Omega=\{\langle\varphi\rangle \mid 0 \neq \varphi \in \mathbb{C}\}$. The map $\Phi: \mathbb{F}_{q^{n}} \mapsto \mathbb{C}$ defined by $\Phi_{b}=\varphi \Leftrightarrow \varphi(1)=b$ is a bijection because all elements of $\mathbb{C}$ different from 0 are not singular. Hence we can define a multiplication $\star$ on $\mathbb{F}_{q^{n}}$ by $x \star y=\Phi_{y}(x)$ for all $x, y \in \mathbb{F}_{q^{n}}$. Then $S(\Omega)=\left(\mathbb{F}_{q^{n}},+, \star\right)$ is a presemifield such that $(\lambda x) \star y=\lambda(x \star y)$, for all $\lambda \in \mathbb{F}_{q}$, and for all $x, y \in \mathbb{F}_{q^{n}}$ (for more details see, e.g., [25]). If we put

$$
\begin{aligned}
X(\infty) & =\left\{(0, y) \mid y \in \mathbb{F}_{q^{n}}\right\}, \\
X(b) & =\left\{(x, x \star b) \mid x \in \mathbb{F}_{q^{n}}\right\}, \\
\mathfrak{S}(\Omega) & =\left\{X(b) \mid b \in \mathbb{F}_{q^{n}}\right\} \cup\{X(\infty)\},
\end{aligned}
$$

then all the elements of $\mathfrak{S}(\Omega)$ are $\mathbb{F}_{q^{-}}$-subspaces of $\mathbb{F}_{q^{n}} \times \mathbb{F}_{q^{n}}$, and $\mathfrak{S}(\Omega)$ is a semifield spread of $\mathrm{PG}(2 n-1, q)=\mathrm{PG}\left(\mathbb{F}_{q^{n}} \times \mathbb{F}_{q^{n}}, \mathbb{F}_{q}\right)$.

All semifield spreads of $\mathrm{PG}(2 n-1, q)$ can be constructed in such a way from a geometric spread set. Isomorphic semifield spreads produce geometric spread sets which are equivalent under the action of the subgroup of $P \Gamma L\left(n^{2}, q\right)$ fixing the rulings of $S_{n, n}$, and conversely (see [21] for $n=2$ and [17] for $n>2$ ).

\subsection{Generalized translation dual of a semifield}

Let $\mathcal{R}$ be any fixed ruling of the Segre variety $S_{n, n}$.

If $X_{1}, \ldots, X_{n-1}$ are elements of $\mathcal{R}$ the subspace $\left\langle X_{1}, \ldots, X_{n-1}\right\rangle$ has dimension $\leq n^{2}-n-1$. Let $\mathcal{T}$ be the set of all subspaces $M=\left\langle X_{1}, \ldots, X_{n-1}\right\rangle$ such that 
$\operatorname{dim} M=n^{2}-n-1$. Then $M$ is contained in $\mathcal{M}\left(S_{n, n}\right)$ and $\mathcal{M}\left(\mathcal{S}_{n, n}\right)=\bigcup_{M \in \mathcal{T}} M$ (see [26]).

If $\mathbf{a}=\left(a_{0}, a_{1}, \ldots, a_{n-1}\right)$, denote by $\varphi_{\mathbf{a}}$ the element of $E$ defined as

$$
\varphi_{\mathbf{a}}: x \mapsto a_{0} x+a_{1} x^{q}+\cdots+a_{n-1} x^{q^{n-1}}
$$

and let $\perp$ be the polarity of $\mathrm{PG}\left(n^{2}-1, q\right)$ associated with the symmetric nonsingular bilinear form of $E$ defined by $\left\langle\varphi_{\mathbf{a}}, \varphi_{\mathbf{b}}\right\rangle=\operatorname{Tr}_{\mathbb{F}_{q}}(\mathbf{a} \cdot \mathbf{b})$. We have the following results.

Theorem 4.1 (Lunardon, Marino, Polverino, Trombetti [26]). An element $X$ belongs to $\mathcal{R}$ if and only if $X^{\perp} \in \mathcal{T}$.

Theorem 4.2 (Lunardon, Marino, Polverino, Trombetti [26]). Let $T$ be a $(2 n-1)$ dimensional subspace of $\mathrm{PG}\left(n^{2}-1, q\right)$ satisfying the following conditions:

$\left(T_{1}\right) T \cap T^{\perp}=\emptyset$;

$\left(T_{2}\right) T^{\perp} \cap \mathcal{S}_{n, n}=\emptyset$;

$\left(T_{3}\right)\left\langle X, T^{\perp}\right\rangle \cap T \subset \mathcal{M}\left(\mathcal{S}_{n, n}\right) \quad \forall X \in \mathcal{R}$.

Then, for each geometric spread set $\Omega$ contained in $T$, the set $\Omega_{T}^{*}=\Omega^{*} \cap T$, with $\Omega^{*}$ the dual of $\Omega$, turns out to be a geometric spread set as well.

The semifield spread associated with $\Omega_{T}^{*}$ is called the translation dual (with respect to $T$ ) of the semifield spread associated with $\Omega$. Moreover we have the following theorem.

Theorem 4.3 (Lunardon, Marino, Polverino, Trombetti [26]). Two semifield spreads of $\mathrm{PG}(2 n-1, q)$ are isomorphic if and only if their translation duals are.

\subsection{Examples}

\section{The classical translation dual}

If $n=2$, then $\operatorname{PG}\left(E, \mathbb{F}_{q}\right)=\operatorname{PG}(3, q)$ and the whole space satisfies Properties $\left(T_{1}\right),\left(T_{2}\right)$ and $\left(T_{3}\right)$ of Theorem 4.2. Also, $S_{2,2}=Q^{+}(3, q)$ is a hyperbolic quadric and $\mathcal{M}\left(S_{n, n}\right)=Q^{+}(3, q)$. Hence if $n=2$, the construction described in Theorem 4.2 returns the classical translation dual operation (see [26]).

\section{The symplectic dual}

Let $n=3$ and $q$ odd. Let

$$
T=\left\{\left\langle x \in \mathbb{F}_{q^{3}} \mapsto a x+b^{q} x^{q}+b x^{q^{2}} \in \mathbb{F}_{q^{3}}\right\rangle \mid a, b \in \mathbb{F}_{q^{3}},(a, b) \neq(0,0)\right\}
$$


be the 5-dimensional subspace of $\mathrm{PG}\left(\operatorname{End}\left(\mathbb{F}_{q^{3}}, \mathbb{F}_{q}\right), \mathbb{F}_{q}\right)=\operatorname{PG}(8, q)$ defined by the self-adjoint maps with respect to the non-degenerate bilinear form $\langle$,$\rangle .$ Then $\mathcal{V}=T \cap S_{n, n} \subset \mathcal{M}\left(S_{n, n}\right)$ is a Veronesian surface of $T$.

Note that $\Omega$ is a geometric spread set contained in $\mathcal{V}$ if and only if the semifield spread $\mathfrak{S}(\Omega)$ is symplectic.

It is easy to verify that the properties of Theorem 4.2 are satisfied. Hence, if $\Omega$ is a geometric spread set contained in $T$, then $\Omega_{T}^{*}$ is a geometric spread set as well, and the symplectic semifield spread $\mathfrak{S}\left(\Omega_{T}^{*}\right)$ arising from $\Omega_{T}^{*}$ is the symplectic dual of the symplectic semifield spread $\mathfrak{S}(\Omega)$ as constructed in [24].

In [24, Theorem 4], the authors apply such a procedure to a Desarguesian geometric spread set contained in $T$, proving that the symplectic dual of the Galois field $\mathbb{F}_{q^{3}}$ is isotopic to a generalized twisted field. Also in [24] another geometric spread set $\Omega$ contained in $T$ when $q=s^{2}$, is constructed with the following properties: $\Omega$ is a Baer subgeometry of $\operatorname{PG}\left(5, s^{2}\right)[24$, Theorem 1] and the semifield spread associated with $\Omega$ is isomorphic to its symplectic dual $\mathfrak{S}\left(\Omega_{T}^{*}\right)$ [24, Proposition 2].

\section{A special case}

Suppose $n>2$. Let

$$
T=\left\{\left\langle\varphi_{a_{0}, 0, \ldots, 0, a_{h}, 0, \ldots, 0}\right\rangle \mid a_{0}, a_{h} \in \mathbb{F}_{q^{n}},\left(a_{0}, a_{h}\right) \neq(0,0)\right\} .
$$

Then an element $\varphi$ of $E$ belongs to $T^{\perp}$ if and only if $\varphi=\varphi_{0, a_{1}, \ldots, a_{h-1}, 0, a_{h+1} \ldots, a_{n-1}}$ where $\left(a_{1}, \ldots, a_{h-1}, a_{h+1}, \ldots, a_{n-1}\right)$ is different from $(0, \ldots, 0)$. Hence $T \cap$ $T^{\perp}=T^{\perp} \cap \mathcal{S}_{n, n}=\emptyset$.

It has been proved that $\left\langle X, T^{\perp}\right\rangle \cap T \subset \Omega\left(\mathcal{S}_{n, n}\right)$ for all $X \in \mathcal{R}_{i}$ for a given $i \in\{1,2\}$ if and only if either $q$ is even or $q$ is odd and the integer $\frac{n}{\operatorname{gcd}(n, h)}$ is even (see [26]).

It follows that, when $q$ is even or when $q$ is odd and $\frac{n}{g c d(n, h)}$ is even, by applying Theorem 4.3, we can always construct the translation dual of any geometric spread set contained in $T$.

\section{$5 \quad$ Future steps?}

\subsection{A construction}

As many of the reviewed constructions use a polarity, we would like to present an unpublished construction of a commutative semifield which also uses a polarity. 
Let $\omega$ be the polarity of $\mathrm{PG}\left(r-1, q^{n}\right)$ defined by the bilinear form

$$
\left\langle\left(X_{1}, X_{2}, \ldots, X_{r}\right) ;\left(Y_{1}, Y_{2}, \ldots, Y_{r}\right)\right\rangle=X_{1} Y_{1}+X_{2} Y_{2}+\cdots+X_{r} Y_{r}
$$

and let

$$
\Delta=\left\{\left(a, f_{2}(a), \ldots, f_{r}(a)\right) \mid a \in F_{q^{n}}\right\}
$$

be an $\mathbb{F}_{q^{-}}$-linear set of rank $n$. Define a multiplication $\circ$ on $\mathbb{F}_{q^{n}}$ by

$$
x \circ y=x y+\sum_{i=2}^{r} f_{i}(x) f_{i}(y) .
$$

Theorem 5.1. If $P^{\omega}$ is disjoint from $\Delta$ for any point $P$ in $\Delta$, then $S=\mathbb{F}_{q^{n}}(+, \circ)$ is a commutative presemifield central over $\mathbb{F}_{q}$.

Proof. It is clear that multiplication is commutative and distributive. Hence, to prove that $S$ is a commutative presemifield, we only have to prove that $x \circ y=0$ if and only if $x=0$ or $y=0$.

If $x \neq 0, y \neq 0$ and $x \circ y=0$, then the point $\left(y, f_{2}(y), \ldots, f_{r}(y)\right)$ of $\Delta$ belongs to the hyperplane $P^{\omega}$ where $P=\left(x, f_{2}(x), \ldots, f_{r}(x)\right) \in \Delta$. As this is impossible we have proved that $S$ is a presemifield.

As $(\lambda x) \circ y=\lambda(x \circ y)$ for all $\lambda \in \mathbb{F}_{q}$ and $x, y \in \mathbb{F}_{q^{n}}$, we conclude that $S$ is central over $\mathbb{F}_{q}$.

Unfortunately, the only example we know of the above construction is the commutative presemifield, isotopic to a field, defined by

$$
x \circ y=x y-c x^{q} y^{q}
$$

where $c$ has norm (over $\mathbb{F}_{q}$ ) different from 1 . If $-c=\xi^{2}$, then the $\mathbb{F}_{q}$-linear set $\Delta=\left\{\left(a, \xi a^{q}\right) \mid a \in \mathbb{F}_{q^{n}}\right\}$ has the required properties.

\subsection{Open problems}

We conclude by listing some open problems:

- The original main problem in the theory of semifield flocks: construct a new semifield flock or prove that new ones do not exist!

- Only one class of examples of commutative semifields of even order is actually known (see [12]). Therefore it would be interesting to construct some new commutative semifields of even order. 
- In order to construct some new examples of symplectic semifield spreads of $\operatorname{PG}(5, q), q$ even, one might extend the construction of the symplectic dual to the even case.

- In $\mathrm{PG}(8, q)$ classify all subspaces $T$ of dimension 5 which satisfy the conditions of Theorem 4.2.

- Determine the generalized translation dual of all known commutative semifields.

\section{References}

[1] L. Bader, G. Lunardon and I. Pinneri, A new semifield flock, J. Combin. Theory Ser. A 86 (1999), 49-62.

[2] A. Blokhuis and G.E. Moorhouse, Some $p$-ranks related to orthogonal spaces, J. Algebraic Combin. 17 (1997), 31-47.

[3] I. Cardinali, G. Lunardon, O. Polverino and R. Trombetti, Spreads in $H(q)$ and 1-systems of $Q(6, q)$, European J. Combin. 23 (2002) 367-376.

[4] J.P. Conway, P.B. Kleidman and R.A. Wilson, New families of ovoids in $\mathrm{O}_{8}^{+}$, Geom. Dedicata 26 (1986), 97-122.

[5] P. Dembowski, Finite Geometries, Springer-Verlag, Berlin Heidelberg New York, 1968.

[6] J.C. Fisher and J.A. Thas, Flocks in PG(3, q), Math. Z. 169 (1979), 1-11.

[7] M.J. Ganley, Central weak nucleus semifields, European J. Combin. 2 (1981), 339-347.

[8] H. Gevaert and N.L. Johnson, Flocks of quadratic cones, generalized quadrangles and translation planes, Geom. Dedicata 27 (1988), 301-317.

[9] A. Gunawardena and G.E. Moorhouse, The non-existence of ovoids in $O_{9}(q)$, European J. Combin 18 (1997), 171-173.

[10] W.M. Kantor, Ovoids and translation planes, Canad. J. Math. 5 (1982), 1195-1207.

[11] - Some generalized quadrangles with parameters $\left(q^{2}, q\right)$, Math. $Z$. 192 (1986), 45-50.

[12] _ Commutative semifields and symplectic spreads, J. Algebra 270 (2003), 96-114. 
[13] J.W.P. Hirschfeld, Finite projective spaces of three dimensions, Oxford University Press, 1985.

[14] J.W.P. Hirschfeld and J.A. Thas, General Galois geometries, Oxford University Press, Oxford, 1991.

[15] N.L. Johnson, Semifield flocks of quadratic cones, Bull. Belg. Math. Soc. Simon Stevin 61 (1987), 313-326.

[16] N.L. Johnson, V. Jha and M. Biliotti, Handbook of finite translation planes, Chapman \& Hall, 2007.

[17] M. Lavrauw, On the isotopism classes of finite semifields, Finite Fields Appl. 14 (2008), 897-910.

[18] G. Lunardon, Flocks, Ovoids of $Q(4, q)$ and Designs, Geom. Dedicata 66 (1997), 163-173.

[19] _ Normal spreads, Geom. Dedicata 75 (1999), 245-261.

[20] _ L Linear $k$-blocking sets, Combinatorica 21 (2001), 571-581.

[21] _ , Translation ovoids, J. Geom. 76 (2003), 200-215.

[22] _ Projective planes of Lenz-Barlotti class V, J. Geom. 101 (2011), $157-172$.

[23] G. Lunardon and O. Polverino, Translation ovoids of orthogonal polar spaces, Forum Math. 16 (2004), 663-669.

[24] G. Lunardon, G. Marino, O. Polverino and R. Trombetti, Symplectic Semifield Spreads of $\mathrm{PG}(5, q)$ and the Veronese Surface, Ric. Mat. 60 (2011), 125-142.

[25] - Maximum scattered linear sets of pseudoregulus type and the Segre Variety $\mathcal{S}_{n, n}$, J. Algebraic Combin. 39 (2014), 807-831.

[26] (2017), DOI 10.1007/s00026-017-0362-0.

[27] G.E. Moorhouse, Root lattice constructions of ovoids, Finite Geometries and Combinatorics (Deinze 1992), Cambridge University Press 1993, 269275.

[28] S.E. Payne, A new infinite family of generalized quadrangles, Congr. Numer. 49 (1985), 115-128.

[29] S.E. Payne and J.A. Thas, Finite Generalized Quadrangles, Pitman, 1984. 
[30] T. Penttila and B. Williams, Ovoids of parabolic spaces, Geom. Dedicata 82 (2000), 1-19.

[31] J.A. Thas, Ovoids and spreads of finite classical polar spaces, Geom. Dedicata 10 (1981), 135-143.

[32] , Generalized quadrangles and flocks of cones, European J. Combin. 8 (1987), 293-312.

[33] , Generalized quadrangles of order $\left(s, s^{2}\right), I I, J$. Combin. Theory Ser. A 79 (1997), 223-254.

[34] _ Symplectic spreads in $\mathrm{PG}(3, q)$, inversive planes and projective planes, Discrete Math. 174 (1997), 329-336.

[35] J.A. Thas and S.E. Payne, Spreads and ovoids in finite generalized quadrangles. Geom. Dedicata 52 (1994), 227-253.

[36] J.A. Thas, K. Thas and H. Van Maldeghem, Translation generalized quadrangles, World Scientific, 2006.

[37] M. Walker, A class of translation planes, Geom. Dedicata 5 (1976), 135146.

[38] C. Weibel, Survey of non-Desarguesian planes, Notices Amer. Math. Soc. 54 (2007), 1294-1302.

Guglielmo Lunardon

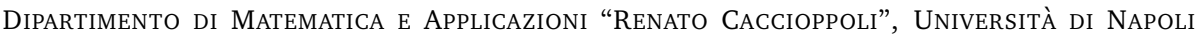
"Federico II", Complesso di Monte S. Angelo, Edificio 5A, Via Cintia, I-80126 Napoli, ITALY., TEL.: +39-081-675602

e-mail: lunardon@unina.it 